University of Nebraska - Lincoln

DigitalCommons@University of Nebraska - Lincoln

Faculty Publications from the Department of Engineering Mechanics

Mechanical \& Materials Engineering,

Department of

December 1996

\title{
Diffuse energy propagation on heterogeneous plates: Structural acoustics radiative transfer theory
}

Joseph A. Turner

University of Nebraska - Lincoln, jaturner@unl.edu

Richard L. Weaver

University of Illinois at Urbana-Champaign, Urbana, Illinois

Follow this and additional works at: https://digitalcommons.unl.edu/engineeringmechanicsfacpub

Part of the Mechanical Engineering Commons

Turner, Joseph A. and Weaver, Richard L., "Diffuse energy propagation on heterogeneous plates: Structural acoustics radiative transfer theory" (1996). Faculty Publications from the Department of Engineering Mechanics. 7.

https://digitalcommons.unl.edu/engineeringmechanicsfacpub/7

This Article is brought to you for free and open access by the Mechanical \& Materials Engineering, Department of at DigitalCommons@University of Nebraska - Lincoln. It has been accepted for inclusion in Faculty Publications from the Department of Engineering Mechanics by an authorized administrator of DigitalCommons@University of Nebraska - Lincoln. 


\title{
Diffuse energy propagation on heterogeneous plates: Structural acoustics radiative transfer theory
}

\author{
Joseph A. Turner ${ }^{\text {a) }}$ and Richard L. Weaver \\ Department of Theoretical and Applied Mechanics, 216 Talbot Laboratory, 104 South Wright Street, \\ University of Illinois at Urbana-Champaign, Urbana, Illinois 61801
}

(Received 2 January 1996; accepted for publication 9 July 1996)

\begin{abstract}
The propagation of diffuse energy on an unwetted flat plate with attached heterogeneities is examined using a statistical, multiple scattering approach. The statistically homogeneous heterogeneities lightly couple the membrane and flexural waves. The problem is formulated in terms of the Bethe-Salpeter equation, which governs the field covariance. It is reduced to a radiative transfer equation in the limit that the attenuations per wave number are small, i.e., when the heterogeneities are weak. This radiative transfer equation governs the diffuse energy propagation as a function of space, time, and propagation direction. Solutions of the radiative transfer equation are presented for the simple case of attached heterogeneities in the form of delta-correlated springs excited by an extensional point source. The results show the evolution of the extensional, shear, and flexural energy densities across the plate as a function of time. A similar approach is expected to apply to the more complicated case of submerged complex structures. (C) 1996 Acoustical Society of America.
\end{abstract}

PACS numbers: 43.40.At, 43.40.Dx [CBB]

\section{INTRODUCTION}

Numerous difficulties are often associated with the analysis of wave propagation on complex submerged shells. Heterogeneities attached to the shell typically scatter the energy propagating on the main structure. The scattered energy subsequently propagates into the surrounding fluid, into the internal structure, and into other propagation modes. The description of the energy propagation on such structures is thus not trivial.

Complicated structures have three frequency regimes which are distinguished for purposes of simplifying analysis. In the low-frequency regime, local heterogeneities are much smaller than a wavelength and have little effect on responses. Finite element analyses are successfully used because the structure can be accurately described by its lowest few modes. In the high-frequency regime, energy deposited on the shell surface is quickly shed back into the fluid before much scattering can occur. Here, ray methods are particularly successful. In the intermediate frequency range, scattering effects are important and must be included in any description of the shell response. In this regime finite element methods fail because of the high number of modes necessary to model the structure accurately. For this reason, statistical methods have been developed to describe energy propagation in this complicated frequency regime.

Statistical approaches to the vibration and acoustics of complex systems are not new. Statistical energy analysis (SEA) ${ }^{1}$ which has its roots in the $1960 \mathrm{~s},{ }^{2,3}$ was perhaps the first rational approach for the examination of the ensemble average vibrational behavior of such structures. The energy flow through a structure is modeled by a steady-state heat

\footnotetext{
${ }^{a)}$ Current address: Fraunhofer Institute for Nondestructive Testing (IzfP), Dept. GR., Building 37, University, 66123 Saarbrücken, Germany.
}

conduction (diffusion) equation. The validity of SEA is based on the assumptions that the fields within substructures are fully equipartitioned. The energy is then assumed to diffuse between substructures. In order to satisfy the SEA assumptions each substructure must usually be highly reverberant with many excited modes. SEA has been used most successfully to obtain average energy distribution information in the steady state. The range of applicability of SEA has been the subject of considerable research and debate. Langley ${ }^{4,5}$ recently developed a wave intensity analysis (WIA) technique which relaxes the isotropy of energy assumption required by SEA. Essentially, he expanded the energy density angular distribution in terms of a finite Fourier series. The first term of his expansion is then the SEA result. Higher-order Fourier components are corrections to the SEA solution. Langley still used the steady-state diffusion equation model although he recognized its limitations for highly damped structures and for describing behavior near sources. Diffuse fields have also been discussed by Weaver regarding unwetted flat plates, ${ }^{6,7}$ elastic half spaces, ${ }^{8}$ and submerged thin shells. ${ }^{9}$

In this article, which expands on previous work, ${ }^{10}$ a statistical approach is used as well. The attached heterogeneities are assumed to couple the membrane and flexural waves lightly. The diffuse plate energy propagation is examined using a multiple scattering approach. The problem is formulated in terms of the Bethe-Salpeter equation which governs the field covariance. The Bethe-Salpeter equation is then reduced to an equation of radiative transfer in the limit that the heterogeneities are weak. The derived structural acoustics radiative transfer equation (SARTE) is the main result presented. This SARTE governs the propagation of the specific intensities of the diffuse extensional, shear, and flexural wave types as a function of space, time, and propagation direction. The diffuse fields in this case are the result of 
scattering from random heterogeneities. This is in contrast to the usual case in reverberation room acoustics and SEA in which the incoherence follows from irregular reverberant reflections from the boundaries of substructures. For early times, locations near sources, or for highly damped systems, the SARTE reduces to a single scattering equation. At late times, or far from sources, the radiative transfer equation reduces to a time-dependent diffusion equation. The main assumption used in the derivation of the SARTE, that the heterogeneities are weak, may limit the range of applicability. However, the scatterers are only assumed weak in the sense that mean-free propagation paths are long compared to a wavelength. This aspect is more extensively addressed in the body of the article.

Rybak used a similar approach to describe the onedimensional steady-state propagation of weakly coupled flexural and longitudinal energy. ${ }^{11,12}$ However, he reduced the Bethe-Salpeter equation to a steady-state diffusion equation directly without the intermediate radiative transfer step. It is the radiative transfer equation with retained time dependence and shear modes that we suggest sheds new light on the study of complex shells.

A brief clarification is also made at this time. The SARTE describes the energy propagation in terms of a quantity called specific intensity. It is defined as the energy flux per unit time per planar angle and is a scalar quantity. Langley uses the term intensity in his work as well., ${ }^{4,5}$ However, his intensity is a vector quantity which is identified here as the energy flux vector.

In Sec. I the problem of interest is outlined and pertinent quantities defined. Section II contains a discussion of the Bethe-Salpeter equation which governs the energy density. The Bethe-Salpeter equation is reduced to a radiative transfer equation in Sec. III. In Sec. IV a numerical solution method is discussed. Finally, results for the simple case of delta-correlated springs are presented in Sec. V.

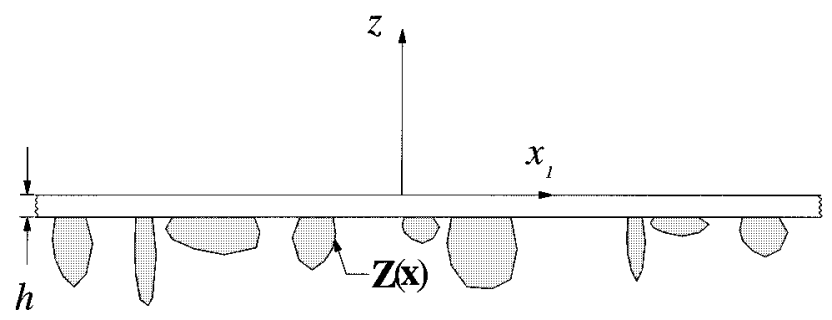

FIG. 1. Infinite flat plate with added spatially varying impedance, $Z(x)$.

\section{STOCHASTIC PLATE EQUATION}

Consider an unwetted flat plate with thickness $h$ as shown in Fig. 1. The plate has in-plane displacements $u_{1}$ and $u_{2}$ and out-of-plane displacement $w$ in the $z$ direction. Random spatially varying complex impedances are attached such that extensional, shear, and flexural motions are all coupled. The added impedances are assumed to have spatial distribution and coupling strengths with known first- and secondorder statistics.

The motion of the plate is described by the Green's dyadic $G_{i j}\left(\mathbf{x}, \mathbf{x}^{\prime} ; t\right)$ which defines the $i$ th Cartesian displacement response at $\mathbf{x}$ due to an excitation in the $j$ th Cartesian direction $(i, j=1,2,3)$ applied at $\mathbf{x}^{\prime}$, where the vector $\mathbf{x}$ is a twodimensional vector $\left(x_{1}, x_{2}\right)$ defined within the plane of the plate. The temporal Fourier transform pair of $\mathbf{G}$ is defined as

$$
\begin{aligned}
& G_{i j}\left(\mathbf{x}, \mathbf{x}^{\prime} ; \omega\right)=\int_{0}^{+\infty} G_{i j}\left(\mathbf{x}, \mathbf{x}^{\prime} ; t\right) e^{i \omega t} d t, \\
& G_{i j}\left(\mathbf{x}, \mathbf{x}^{\prime} ; t\right)=\frac{1}{2 \pi} \int_{-\infty}^{+\infty} G_{i j}\left(\mathbf{x}, \mathbf{x}^{\prime} ; \omega\right) e^{-i \omega t} d \omega .
\end{aligned}
$$

The temporally Fourier transformed coupled membraneplate equations for this plate are

$$
\left[Z_{k i}(\mathbf{x}, \omega)+\mathscr{D}_{k i}\right] G_{i j}\left(\mathbf{x}, \mathbf{x}^{\prime} ; \omega\right)=\delta_{k j} \delta^{2}\left(\mathbf{x}^{\prime}-\mathbf{x}\right) .
$$

The plate operator, $\mathscr{L}_{k i}$, is given by

$$
\mathscr{D}=\left[\begin{array}{ccc}
-\frac{\partial^{2}}{\partial x_{1}^{2}}-\frac{1-\nu}{2} \frac{\partial^{2}}{\partial x_{2}^{2}}-\frac{(\omega+i \epsilon)^{2}}{c_{p}^{2}} & -\frac{1+\nu}{2} \frac{\partial^{2}}{\partial x_{1} \partial x_{2}} & 0 \\
-\frac{1+\nu}{2} \frac{\partial^{2}}{\partial x_{1} \partial x_{2}} & -\frac{\partial^{2}}{\partial x_{2}^{2}}-\frac{1-\nu}{2} \frac{\partial^{2}}{\partial x_{1}^{2}}-\frac{(\omega+i \epsilon)^{2}}{c_{p}^{2}} & 0 \\
0 & 0 & \frac{h^{2}}{12} \nabla^{4}-\frac{(\omega+i \epsilon)^{2}}{c_{p}^{2}}
\end{array}\right],
$$

where $c_{p}^{2}=E /\left(1-\nu^{2}\right) \rho$ defines the extensional, or "plate" wave speed and $E$ is the elastic modulus, $\nu$ is the Poisson's ratio, and $\rho$ is the volumetric density of the plate material. The shear and flexural wave speeds are $c_{s}^{2}=(1-\nu) c_{p}^{2} / 2$ and $c_{f}^{2}=h \omega c_{p} / \sqrt{12}$, respectively. The frequency dependence of $c_{f}$ will remain implicit throughout. An infinitesimal imaginary part, $\epsilon$, has been added to the frequency $\omega$ to emphasize that the transform, Eq. (1a), is defined for $\operatorname{Im}\{\omega\}>0$.

The random impedance parameter $Z_{k i}(\mathbf{x}, \omega)$ is a function of position and frequency and couples all propagation modes. It is assumed here to be a local operator. ${ }^{10}$ The fluctuations of the added impedance are assumed small such that the average added impedance, $\mathbf{Z}^{0}=\langle\mathbf{Z}\rangle$, and covariance, $\left\langle\left(\mathbf{Z}(\mathbf{x})-\mathbf{Z}^{0}\right)\left(\mathbf{Z}(\mathbf{y})-\mathbf{Z}^{0}\right)\right\rangle$, are the only statistics of importance. The brackets, \langle\rangle , denote an ensemble average. The covariance of the heterogeneities is further assumed to have the following form:

$$
\left\langle\left(Z_{\alpha \gamma}(\mathbf{x})-Z_{\alpha \gamma}^{0}\right)\left(Z_{\delta \beta}(\mathbf{y})-Z_{\delta \beta}^{0}\right)\right\rangle={ }_{\beta}^{\alpha} \Xi_{\delta}^{\gamma} W(\mathbf{x}-\mathbf{y}) .
$$

Here, $W$ is a spatial two-point correlation function. For the 
form shown in Eq. (4), it has been assumed that the distribution of impedance orientation is independent of the spatial distribution. The assumption of statistical homogeneity is evident in the form of $W$ which is a function of $\mathbf{x}-\mathbf{y}$ only. The fourth-rank tensor $\boldsymbol{\Xi}$ and the function $W$ will, in general, also be functions of inner frequency, $\omega$.

Equation (2) is a stochastic partial differential equation because of the random nature of $\mathbf{Z}$. It has deterministic operator, $\mathscr{L}_{k i}$, characteristic of the decoupled membrane-plate equations. It also has the random operator, $Z_{k i}$. Equation (2) is of the form considered by Frisch ${ }^{13}$ for the propagation of waves through random media. Previously, the mean plate response, $\langle\mathbf{G}\rangle$, was derived by solution of the Dyson equation. ${ }^{10}$ Scattering attenuations due to mode conversion effects were calculated in the limit of weak heterogeneities. Our emphasis here is on the diffuse energy propagation and thus the covariance of the Green's dyadic, $\left\langle\mathbf{G G}^{*}\right\rangle$. This quantity is related to the acoustic energy density ${ }^{4,5,14}$ and is governed by the Bethe-Salpeter equation. ${ }^{13,14}$

An important distinction must also be made regarding the two different time and length scales of this problem. The short time behavior of the plate displacements, when Fourier transformed, becomes a function of the inner frequency, $\omega$, present in the coupled membrane-plate operator, Eq. (3). The much slower time scale associated with the evolving diffuse energy Fourier transforms to an outer frequency, $\Omega$. Similarly, the two length scales of this problem transform to an inner wave vector, $\mathbf{p}$ or $\mathbf{q}$ (with magnitude of order $\omega / c$ ), and an outer wave vector denoted by $\boldsymbol{\Delta}$.

Thus, the spatial Fourier transform of the covariance of the Green's dyadic is properly used to represent the diffuse acoustic energy density, $E$, as has been previously discussed. ${ }^{14}$ The spatially and temporally transformed diffuse energy density is defined as

$$
E(\Omega, \Delta) \equiv \int d^{2} x e^{i \Delta \cdot\left(\mathbf{x}-\mathbf{x}^{\prime}\right)}\left\langle\mathbf{G}_{\omega}\left(\mathbf{x}, \mathbf{x}^{\prime}\right): \mathbf{G}_{\omega+\Omega}^{*}\left(\mathbf{x}, \mathbf{x}^{\prime}\right)\right\rangle,
$$

where the $*$ denotes a complex conjugate and also denotes the Green's dyadic evaluated at $\omega+\Omega$. It has been implicitly assumed in Eq. (5) that the energy has been bandpass filtered over a frequency band, $\Delta \omega$ such that $\omega \gg \Delta \omega \gg \Omega{ }^{14}$ This assumption is equivalent to the assumptions applied in the derivation of optical radiative transfer equations. ${ }^{15,16}$

\section{BETHE-SALPETER EQUATION}

The relationship between the acoustic energy density and the covariance of the Green's dyadic, $\left\langle\mathbf{G G}^{*}\right\rangle$, allows us to proceed with the derivation of an equation that governs this diffuse energy as a function of space, time, and propagation direction.

We begin by defining the spatial Fourier transform of the covariance

$$
\begin{aligned}
{ }_{\mathbf{p}^{\prime} i}^{\mathbf{p} \alpha} H_{j \mathbf{q}^{\prime}}^{\beta \mathbf{q}} & \delta^{2}\left(\mathbf{p}+\mathbf{q}^{\prime}-\mathbf{q}-\mathbf{p}^{\prime}\right) \\
= & \frac{1}{(2 \pi)^{4}} \iiint \int d^{2} x d^{2} x^{\prime} d^{2} y d^{2} y^{\prime}\left\langle G_{\alpha \beta}\left(\mathbf{x}, \mathbf{x}^{\prime}\right)\right. \\
& \left.\times G_{i j}^{*}\left(\mathbf{y}, \mathbf{y}^{\prime}\right)\right\rangle e^{-i \mathbf{p} \cdot \mathbf{x}+i \mathbf{q} \cdot \mathbf{x}^{\prime}+i \mathbf{p}^{\prime} \cdot \mathbf{y}-i \mathbf{q}^{\prime} \cdot \mathbf{y}^{\prime}} .
\end{aligned}
$$

The energy density is given in terms of this quantity by

$$
E=\frac{1}{(2 \pi)^{2}} \iint d^{2} q d^{2} p \underset{\mathbf{p}+\Delta i}{\mathbf{p} \alpha} H_{j \mathbf{q}+\Delta}^{\beta \mathbf{q}} \delta_{i \alpha} \delta_{j \beta} .
$$

The stochastic partial differential equation given by Eq. (2) is of the form discussed extensively by Frisch for wave propagation through random media. ${ }^{13}$ From his treatise, it is clear that there are two fundamental equations necessary for the present work. The Dyson equation governs the mean response, $\langle\mathbf{G}\rangle$, and was considered previously. ${ }^{10}$ There it was shown that attenuations due to scattering arise because of the interaction of the propagating waves with the heterogeneities. These attenuations are not the result of true dissipative processes which would extract energy from the plate. Instead, these scattering attenuations are the result of a loss of coherence of the field due to scattering. One particular wave type propagating in a given direction will scatter its energy to other propagation types and other propagation directions. This result will be illustrated here as well.

Here we begin with the Bethe-Salpeter equation, which governs the covariance of the Green's dyadic, $\left\langle\mathbf{G G}^{*}\right\rangle{ }^{13,14}$ In spatial transform space the Bethe-Salpeter equation is

$$
\begin{aligned}
\underset{\mathbf{p}+\Delta i}{\mathbf{p} \alpha} H_{j \mathbf{q}^{+} \Delta}^{\beta \mathbf{q}}= & { }_{i}^{\alpha} \Gamma_{j \mathbf{p}+\Delta}^{\beta \mathbf{p}} \delta^{2}(\mathbf{p}-\mathbf{q}) \\
& +\int d^{2} s_{i}^{\alpha} \Gamma_{k \mathbf{p}+\Delta}^{\gamma \mathbf{p}} \underset{\mathbf{p}+\Delta k}{\mathbf{p} \gamma} K_{l \mathbf{s}+\Delta}^{\delta \mathbf{s}} \stackrel{\mathbf{s} \delta}{\mathbf{s}+\Delta l} H_{j \mathbf{q}+\Delta}^{\beta \mathbf{q}},
\end{aligned}
$$

where

$$
{ }_{i}^{\alpha} \Gamma_{k \mathbf{p}+\Delta}^{\gamma \mathbf{p}} \equiv\left\langle G_{\alpha \gamma}(\mathbf{p})\right\rangle\left\langle G_{i k}^{*}(\mathbf{p}+\Delta)\right\rangle,
$$

is the double mean Green's dyadic. The intensity operator, $\mathbf{K}$, describes the interaction of the energy with the heterogeneities. Like the Dyson equation, ${ }^{10}$ the Bethe-Salpeter equation is exact and includes all multiple scattering effects. However, the Bethe-Salpeter equation is a true integral equation in the sense that a spatial transform does not simplify the integral nature of the equation as is the case with the Dyson equation. ${ }^{10,13}$ Tractable solutions are often realized only by approximation.

The results for the mean plate response were derived assuming that the heterogeneities were not large, i.e., that the attenuations per wave number were small. ${ }^{10}$ This same approximation is used here as well. The Keller approximation, ${ }^{17}$ also called the first-order smoothing approximation ${ }^{13}$ (FOSA) or Bourret approximation, ${ }^{6,7}$ allows the intensity operator, $\mathbf{K}$, to be approximated as ${ }^{13,14}$

$$
\underset{\mathbf{p} \gamma+\Delta k}{\mathbf{p} \gamma} K_{l \mathbf{s}+\Delta}^{\delta \mathbf{s}} \cong{ }_{k}^{\gamma} \Xi_{l}^{\delta} \widetilde{W}(\mathbf{p}-\mathbf{s}) .
$$

In Eq. (10), $\widetilde{W}$ is the spatial Fourier transform of the twopoint correlation function discussed above. The assumption of statistical homogeneity appears in $\widetilde{W}$ which is a function 
of $\mathbf{p}-\mathbf{s}$ rather than the individual wave numbers, $\mathbf{p}$ and $\mathbf{s}$. The fourth-rank tensor $\Xi$ is in general complex if the heterogeneities have internal losses. This approximation is often termed the "ladder approximation" in reference to the shapes of the associated diagrams which can be used to represent $\mathbf{H}$.

The ladder approximation implies a limitation on the range of validity of the subsequent analysis. It has been assumed that the scattering is weak such that the scattering attenuation per wave number, $\alpha / k$, is small. The quantity $\alpha / k$ is a critical parameter in localization studies. In fact $\alpha / k \sim 1$ is the Ioffe-Regel criterion for the onset of Anderson localization. ${ }^{18,19}$ Thus, the use of the ladder approximation precludes any prediction of localization. However, the criterion $\alpha \ll k$ is generally not very restrictive for most applications.

A somewhat simpler form of the Bethe-Salpeter equation is obtained by definition of a new quantity, $\mathbf{S}$, which defines the source of the covariance

$$
S_{m}^{\mu}(\mathbf{p}, \Delta) \equiv \int d^{2} q_{m}^{\mu}\left(\Gamma^{-1}\right)_{i \mathbf{p}+\Delta}^{\alpha \mathbf{p}} \underset{\mathbf{p}+\Delta i}{\mathbf{p} \alpha} H_{j \mathbf{q}+\Delta}^{\beta \mathbf{q}} \delta_{\beta j} .
$$

In Eq. (11), the inverse double mean Green's dyadic, $\boldsymbol{\Gamma}^{-1}$, is defined as

$$
{ }_{m}^{\mu}\left(\Gamma^{-1}\right)_{i \mathbf{p}+\Delta}^{\alpha \mathbf{p}} \equiv\langle G(\mathbf{p})\rangle_{\mu \alpha}^{-1}\left\langle G^{*}(\mathbf{p}+\Delta)\right\rangle_{m i}^{-1} .
$$

The energy density is given in terms of the quantity $\mathbf{S}$ by

$$
E(\Omega, \Delta)=\frac{1}{(2 \pi)^{2}} \int d^{2} p_{i}^{\alpha} \Gamma_{m \mathbf{p}+\Delta}^{\mu \mathbf{p}} S_{m}^{\mu}(\mathbf{p}, \Delta) \delta_{i \alpha} .
$$

With the definition of $\mathbf{S}$ given by Eq. (11), the BetheSalpeter equation can be appropriately contracted to the following form:

$$
S_{m}^{\mu}(\mathbf{p}, \Delta)=\delta_{\mu m}+\int d^{2}{ }_{\underset{\mathbf{p}}{\mathbf{p}} \mu}^{\mathbf{p} \mu m} K_{j \mathbf{s}+\Delta}^{\alpha \mathbf{s}}{ }_{j}^{\alpha} \Gamma_{l \mathbf{s}+\Delta}^{\delta \mathbf{s}} S_{l}^{\delta}(\mathbf{s}, \Delta) .
$$

As noted previously, ${ }^{14}$ scattering problems such as this one have three naturally arising length scales. The inner and outer wave numbers are $\omega / c$ and $\Delta$, respectively, and the attenuation is $\alpha$. The assumption of weak heterogeneities implies that $\alpha \ll \omega / c$ as was used previously. ${ }^{10}$ The weak heterogeneity assumption also implies that the spatial variation in the diffuse energy varies much slower than the inner wave number, $\Delta \ll \omega / c$. In the next section these two limits are used to reduce the contracted version of Bethe-Salpeter equation, Eq. (14), to a radiative transfer equation. The more restrictive assumption that $\Delta \ll \alpha$ can be used to further reduce the radiative transfer equation to a diffusion equation. ${ }^{14}$

\section{STRUCTURAL ACOUSTICS RADIATIVE TRANSFER}

The Bethe-Salpeter equation, Eq. (14), governs the propagation of the energy density. It is an integral equation without general analytical solutions. However, the integral over the magnitude of the wave number can be simplified within the context of the assumption of weak heterogeneities. The poles of $\boldsymbol{\Gamma}$, defined in Eq. (9) as the double mean
Green's dyadic, will dominate the integral of Eq. (14). ${ }^{10}$ In the limit that $\alpha \ll \omega / c$ and $\Delta \ll \omega / c, s \Gamma$ can be equivalently replaced by the following:

$$
\begin{aligned}
s_{j}^{\alpha} \Gamma_{l \mathbf{s}+\Delta}^{\delta \mathbf{s}} \cong & R_{e}(\hat{\mathbf{s}}, \Delta) \hat{s}_{\alpha} \hat{s}_{\delta} \hat{s}_{j} \hat{s}_{l} \delta\left(|\mathbf{s}|-p_{e}^{0}\right)+R_{s}(\hat{\mathbf{s}}, \Delta) \\
& \times\left(\delta_{\alpha \delta}-\hat{s}_{\alpha} \hat{s}_{\delta}\right)\left(\delta_{j l}-\hat{s}_{j} \hat{s}_{l}\right) \delta\left(|\mathbf{s}|-p_{s}^{0}\right) \\
& +R_{f}(\hat{\mathbf{s}}, \Delta) \hat{z}_{\alpha} \hat{z}_{\delta} \hat{z}_{j} \hat{z}_{l} \delta\left(|\mathbf{s}|-p_{f}^{0}\right),
\end{aligned}
$$

where $\delta_{i j}$ is the two-dimensional Kronecker delta and $\hat{\mathbf{s}}$ is, of course, in the $\left(x_{1}, x_{2}\right)$ plane. The inner wave numbers of the bare plate are defined by

$$
p_{e}^{0}=\omega / c_{p}, p_{s}^{0}=\omega / c_{s}, p_{f}^{0}=\omega / c_{f} .
$$

The extensional, shear, and flexural energy propagators, $R$, are defined in terms of the outer frequency, $\Omega$, and outer wave number, $\Delta$, by

$$
\begin{aligned}
& R_{e}(\hat{\mathbf{s}}, \Delta)=\frac{\pi c_{p} / 2 \omega}{\kappa_{e}-i \hat{\mathbf{s}} \cdot \Delta+i \Omega / c_{p}}, \\
& R_{s}(\hat{\mathbf{s}}, \Delta)=\frac{2 \pi c_{s} / \omega(1-\nu)^{2}}{\kappa_{s}-i \hat{\mathbf{s}} \cdot \Delta+i \Omega / c_{s}}, \\
& R_{f}(\hat{\mathbf{s}}, \Delta)=\frac{2 \pi\left(c_{f} / \omega\right)^{5}}{\left(4 h^{2} / 12\right)^{2}\left[\kappa_{f}-i \hat{\mathbf{s}} \cdot \Delta+i \Omega / 2 c_{f}\right]} .
\end{aligned}
$$

The energy attenuations, $\kappa$, resulting from scattering, are twice the corresponding displacement attenuations, $\alpha .{ }^{10}$ The energy propagators contain the group speeds for the respective propagation modes, as expected.

With the approximation given by Eq. (15), Eq. (14) now becomes

$$
\begin{aligned}
S_{m}^{\mu}(\mathbf{p}, \Delta)= & \delta_{\mu m}+\int_{0}^{2 \pi} d \hat{s}\left\{\begin{array}{l}
\mathbf{p} \mu \\
\mathbf{p} m
\end{array} K_{j \hat{\mathbf{s}} \omega / c_{p}}^{\alpha \hat{\mathbf{s}} \omega / c_{p}} \hat{s}_{\alpha} \hat{s}_{\delta} \hat{s}_{j} \hat{s}_{l} S_{l}^{\delta}(\mathbf{s}, \Delta)\right. \\
& \times R_{e}(\hat{\mathbf{s}}, \Delta)+{ }_{\mathbf{p} m}^{\mathbf{p} \mu} K_{j \hat{j} \omega / c_{s}}^{\alpha \hat{\mathbf{s}} \omega / c_{s}}\left(\delta_{\alpha \delta}-\hat{s}_{\alpha} \hat{s}_{\delta}\right) \\
& \times\left(\delta_{j l}-\hat{s}_{j} \hat{s}_{l}\right) S_{l}^{\delta}(\mathbf{s}, \Delta) R_{s}(\hat{\mathbf{s}}, \Delta) \\
& \left.+{ }_{\mathbf{p} m}^{\mathbf{p} \mu} K_{3 \hat{\mathbf{s}} \omega / c_{f}}^{3 \hat{\hat{\mathbf{s}} \omega / c_{f}}} S_{3}^{3}(\mathbf{s}, \Delta) R_{f}(\hat{\mathbf{s}}, \Delta)\right\}
\end{aligned}
$$

Equation (18) is a radiative transfer-type equation. The source of covariance, $\mathbf{S}$, is equal to the primary source plus a secondary source. The secondary source, in scattered direction $\hat{\mathbf{p}}$, is given by an integral over all incident directions $\hat{\mathbf{s}}$. This integral describes the energy that has scattered from other directions $\hat{\mathbf{s}}$ into direction $\hat{\mathbf{p}}$.

Using the same approximation, Eq. (15), the energy density becomes, in direct notation,

$$
\begin{aligned}
& E(\Omega, \Delta)=\frac{1}{(2 \pi)^{2}} \int_{0}^{2 \pi} d \hat{p}\left\{\mathbf{I}_{\cdot \hat{\mathbf{p}} \hat{\mathbf{p}}}^{\cdot \hat{\mathbf{p}}} \cdot \mathbf{S}\left(\hat{\mathbf{p}} p_{e}^{0}, \Delta\right) R_{e}(\hat{\mathbf{p}}, \Delta)\right.
\end{aligned}
$$

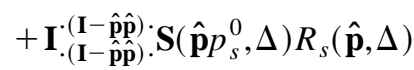

$$
\begin{aligned}
& \left.+{ }_{\hat{\mathbf{z}}}^{\hat{\mathbf{z}}} \cdot \mathbf{S}\left(\hat{\mathbf{p}} p_{f}^{0}, \Delta\right) R_{f}(\hat{\mathbf{p}}, \Delta)\right\},
\end{aligned}
$$

where $\mathbf{I}$ is the two-dimensional identity dyadic $\left(\mathbf{I}=\hat{\mathbf{x}}_{1} \hat{\mathbf{x}}_{1}+\hat{\mathbf{x}}_{2} \hat{\mathbf{x}}_{2}\right)$. 


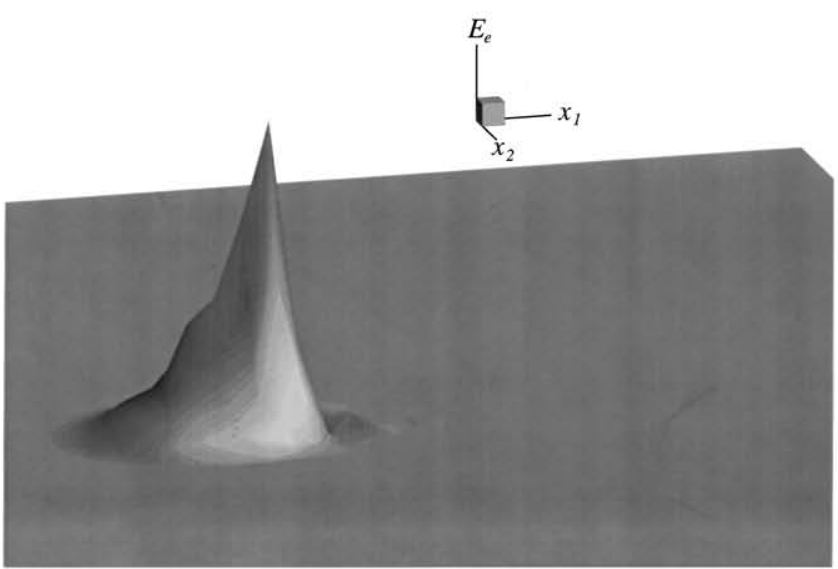

(a)

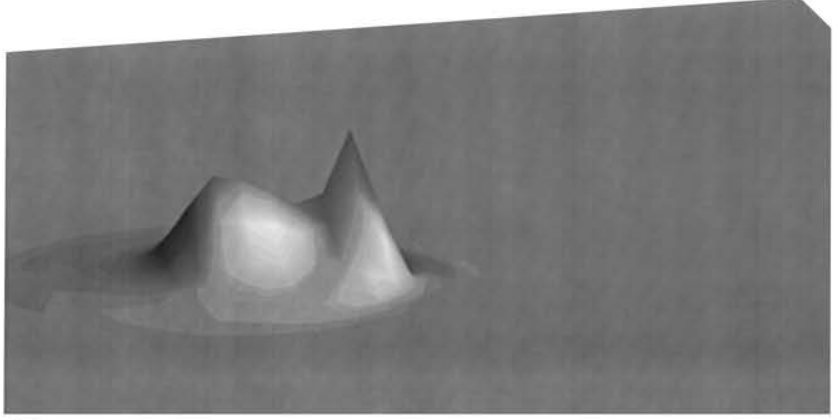

(b)

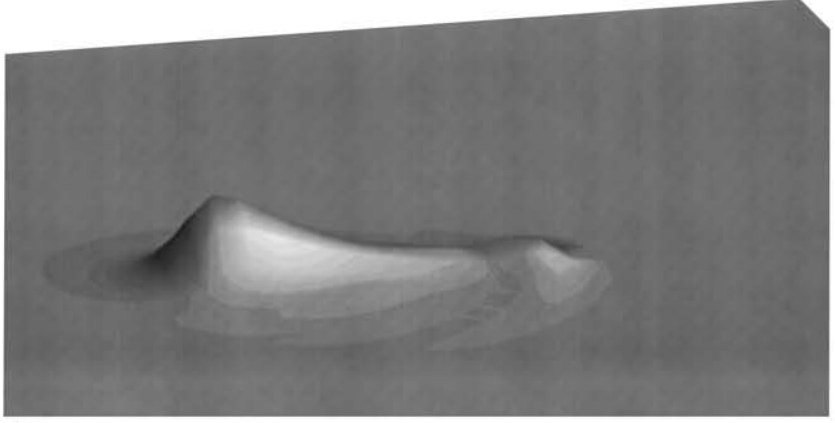

(c)

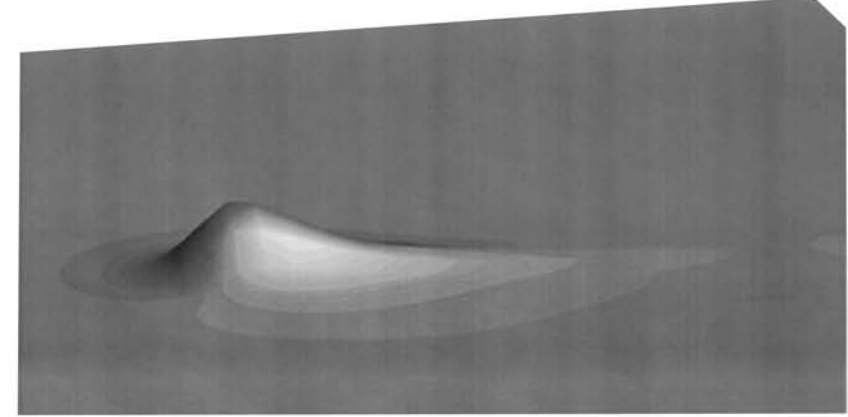

(d)

FIG. 2. Diffuse extensional energy density, $E_{e}$, at $\tilde{t}=1,2,4,7$ extensional mean-free times, respectively (a)-(d), to an incident extensional wave launched in the $\hat{x}_{1}$ direction.

Equation (18) can also be manipulated into a more familiar form of radiative transfer equation. ${ }^{15,16,20}$ First, the source function is expanded in terms of its three nonzero components

$$
\mathbf{S}(\mathbf{p})=\hat{\mathbf{p}} \hat{\mathbf{p}} S_{e}(\hat{\mathbf{p}})+(\mathbf{I}-\hat{\mathbf{p}} \hat{\mathbf{p}}) S_{s}(\hat{\mathbf{p}})+\hat{\mathbf{z}} \hat{\mathbf{z}} S_{f}(\hat{\mathbf{p}}) .
$$

The other components of $\mathbf{S}$ do not contribute to the energy density, Eq. (19). These components describe the coherent interference between the three wave types. Since each wave type has a very different wave speed, such coherencies become unimportant after a propagation length on the order of the wavelength. These components of $\mathbf{S}$ therefore do not appear.

Specific intensities are defined in terms of the source function as

$$
I_{e}=S_{e} R_{e} c_{p}, I_{s}=S_{s} R_{s} c_{s}, I_{f}=S_{f} R_{f} 2 c_{f} .
$$

The specific intensities correspond to the energy flux per unit time per planar angle. They describe the energy density propagating in a particular direction.

The definition of the source function, these specific intensities, and the inverse temporal and spatial transforms of the propagators, Eqs. (17), allow Eq. (18) to be rewritten in its most familiar form ${ }^{20}$

$$
\begin{gathered}
\boldsymbol{\nabla} \cdot \hat{\mathbf{p}} \underline{I}(\mathbf{r}, t, \phi)+\underline{\underline{c}}^{-1} \frac{\partial \underline{I}(\mathbf{r}, t, \phi)}{\partial t}+\underline{I}(\mathbf{r}, t, \phi) \\
=\int_{0}^{2 \pi} \underline{\underline{P}}\left(\boldsymbol{\phi}, \phi^{\prime}\right) \underline{I}\left(\mathbf{r}, t, \phi^{\prime}\right) d \phi^{\prime},
\end{gathered}
$$

where $\phi$ and $\phi^{\prime}$ are now used to represent the propagation directions $\hat{\mathbf{p}}$ and $\hat{\mathbf{s}}$ of Eq. (18). The Stokes vector, $\underline{I}$, is a column vector containing the three Stokes parameters defined in Eq. (21), $\underline{I}=\left\{I_{e}, I_{s}, I_{f}\right\}^{T}$. The group speed and scattering attenuation matrices are

$$
\underline{\underline{c}}=\left[\begin{array}{ccc}
c_{e} & 0 & 0 \\
0 & c_{s} & 0 \\
0 & 0 & 2 c_{f}
\end{array}\right], \underline{\underline{\kappa}}=\left[\begin{array}{ccc}
\kappa_{e} & 0 & 0 \\
0 & \kappa_{s} & 0 \\
0 & 0 & \kappa_{f}
\end{array}\right] .
$$

Note that there are no interference terms as in the electromagnetic or elastic cases. ${ }^{15,16,20}$ This is due to the disparate wave speeds of all wave types. The structural acoustics radiative transfer equation (SARTE) given by Eq. (22) defines the propagation and scattering of flexural and membrane energy as a function of space, time, and propagation direction. It includes all multiple scattering effects and is valid within the limits of its primary assumption that the scattering is weak, before the onset of localization. Radiative transfer equations have been studied extensively for astrophysical and electromagnetic,,${ }^{15,16}$ heat transfer ${ }^{21}$ and ultrasonic applications. ${ }^{20}$

The scattering matrix, $\underline{\underline{P}}\left(\phi, \phi^{\prime}\right)$, has components

$$
\begin{aligned}
& P_{11}\left(\phi, \phi^{\prime}\right)=\left(\pi c_{p} / 2 \omega\right) \xi_{e e}\left(\phi, \phi^{\prime}\right) \widetilde{W}_{e e}\left(\phi, \phi^{\prime}\right), \\
& P_{12}\left(\phi, \phi^{\prime}\right)=\left(\pi c_{p}^{2} / 2 c_{s} \omega\right) \xi_{e s}\left(\phi, \phi^{\prime}\right) \widetilde{W}_{e s}\left(\phi, \phi^{\prime}\right), \\
& P_{13}\left(\phi, \phi^{\prime}\right)=\left(\pi c_{p}^{2} / 4 c_{f} \omega\right) \xi_{e f}\left(\phi, \phi^{\prime}\right) \widetilde{W}_{e f}\left(\phi, \phi^{\prime}\right), \\
& P_{21}\left(\phi, \phi^{\prime}\right)=\left(\pi c_{p}^{3} / 2 c_{s}^{2} \omega\right) \xi_{s e}\left(\phi, \phi^{\prime}\right) \widetilde{W}_{s e}\left(\phi, \phi^{\prime}\right), \\
& P_{22}\left(\phi, \phi^{\prime}\right)=\left(\pi c_{p}^{4} / 2 c_{s}^{3} \omega\right) \xi_{s s}\left(\phi, \phi^{\prime}\right) \widetilde{W}_{s s}\left(\phi, \phi^{\prime}\right), \\
& P_{23}\left(\phi, \phi^{\prime}\right)=\left(\pi c_{p}^{4} / 4 c_{s}^{2} c_{f} \omega\right) \xi_{s f}\left(\phi, \phi^{\prime}\right) \widetilde{W}_{s f}\left(\phi, \phi^{\prime}\right),
\end{aligned}
$$




$$
\begin{aligned}
& P_{31}\left(\phi, \phi^{\prime}\right)=\left(\pi c_{p}^{3} / 4 c_{f}^{2} \omega\right) \xi_{f e}\left(\phi, \phi^{\prime}\right) \widetilde{W}_{f e}\left(\phi, \phi^{\prime}\right), \\
& P_{32}\left(\phi, \phi^{\prime}\right)=\left(\pi c_{p}^{4} / 4 c_{s} c_{f}^{2} \omega\right) \xi_{f s}\left(\phi, \phi^{\prime}\right) \widetilde{W}_{f s}\left(\phi, \phi^{\prime}\right), \\
& P_{33}\left(\phi, \phi^{\prime}\right)=\left(\pi c_{p}^{4} / 8 c_{f}^{3} \omega\right) \xi_{f f}\left(\phi, \phi^{\prime}\right) \widetilde{W}_{f f}\left(\phi, \phi^{\prime}\right) .
\end{aligned}
$$

These scattering matrix components are defined in terms of a scattering function, $\xi\left(\phi, \phi^{\prime}\right)$, which is an inner product on $\Xi$, and the spatial Fourier transform of a two-point correlation function, $\widetilde{W}\left(\phi, \phi^{\prime}\right) .{ }^{10}$ The required scattering functions are given by

$$
\begin{aligned}
& \xi_{e e}\left(\phi, \phi^{\prime}\right) \equiv \underset{\hat{\mathbf{p}} \cdot}{\hat{\hat{\mathbf{p}}} \cdot \Xi} \cdot \Xi_{\cdot \hat{\mathbf{s}}}^{\cdot \hat{\mathbf{s}}}={ }_{\beta}^{\alpha} \Xi{ }_{\delta}^{\gamma} \hat{p}_{\alpha} \hat{p}_{\beta} \hat{s}_{\gamma} \hat{s}_{\delta}, \\
& \xi_{e s}\left(\phi, \phi^{\prime}\right) \equiv \hat{\hat{\mathbf{p}}} \cdot \hat{\hat{\mathbf{p}}} \cdot \Xi \cdot\left(\mathbf{I}-{ }_{\hat{\mathbf{s}}}^{\hat{\mathbf{s}}}\right)={ }_{\beta}^{\alpha} \Xi{ }_{\delta}^{\gamma} \hat{p}_{\alpha} \hat{p}_{\beta}\left(\delta_{\gamma \delta}-\hat{s}_{\gamma} \hat{s}_{\delta}\right), \\
& \xi_{e f}\left(\phi, \phi^{\prime}\right) \equiv \hat{\hat{\mathbf{p}}} \cdot \hat{\hat{\mathbf{p}}} \cdot \Xi_{\cdot \hat{\mathbf{z}}}^{\cdot \hat{\mathbf{z}}}={ }_{\beta}^{\alpha} \Xi_{3}^{3} \hat{p}_{\alpha} \hat{p}_{\beta}, \\
& \xi_{s e}\left(\phi, \phi^{\prime}\right) \equiv(\mathbf{I}-\hat{\hat{\mathbf{p}}}) \cdot \Xi_{\cdot \hat{\mathbf{s}}}^{\cdot \hat{\mathbf{s}}}={ }_{\beta}^{\alpha} \Xi_{\delta}^{\gamma}\left(\delta_{\alpha \beta}-\hat{p}_{\alpha} \hat{p}_{\beta}\right) \hat{s}_{\gamma} \hat{s}_{\delta}, \\
& \xi_{s s}\left(\phi, \phi^{\prime}\right) \equiv(\mathbf{I}-\hat{\mathbf{p}}) \cdot \Xi \cdot(\mathbf{I}-\hat{\mathbf{s}}) \\
& ={ }_{\beta}^{\alpha} \Xi{ }_{\delta}^{\gamma}\left(\delta_{\alpha \beta}-\hat{p}_{\alpha} \hat{p}_{\beta}\right)\left(\delta_{\gamma \delta}-\hat{s}_{\gamma} \hat{s}_{\delta}\right), \\
& \xi_{s f}\left(\phi, \phi^{\prime}\right) \equiv(\mathbf{I}-\hat{\hat{\mathbf{p}}}) \cdot \Xi_{\cdot \hat{\mathbf{z}}}^{\cdot \hat{\mathbf{z}}}={ }_{\beta}^{\alpha} \Xi_{3}^{3}\left(\delta_{\alpha \beta}-\hat{p}_{\alpha} \hat{p}_{\beta}\right), \\
& \xi_{f s}\left(\phi, \phi^{\prime}\right) \equiv{ }_{\hat{\mathbf{z}}}^{\hat{\mathbf{z}} \cdot} \cdot \Xi \cdot(\mathbf{I}-\hat{\mathbf{s}})={ }_{3}^{3} \Xi{ }_{\delta}^{\gamma}\left(\delta_{\gamma \delta}-\hat{s}_{\gamma} \hat{s}_{\delta}\right), \\
& \xi_{f e}\left(\phi, \phi^{\prime}\right) \equiv_{\hat{\mathbf{z}}}^{\hat{\mathbf{z}} \cdot} \cdot \Xi_{\cdot \hat{\mathbf{s}}}^{\cdot \hat{\mathbf{s}}}={ }_{3}^{3} \Xi{ }_{\delta}^{\gamma} \hat{s}_{\gamma} \hat{s}_{\delta} \\
& \xi_{f f}\left(\phi, \phi^{\prime}\right) \equiv{ }_{\hat{\mathbf{z}}}^{\hat{\mathbf{z}} \cdot} \cdot \Xi_{\cdot \hat{\mathbf{z}}}^{\cdot \hat{\mathbf{z}}}={ }_{3}^{3} \Xi_{3}^{3},
\end{aligned}
$$

with a sum over all indices $\alpha, \beta, \gamma, \delta=1,2$. The unit vectors $\hat{\mathbf{s}}$ and $\hat{\mathbf{p}}$ are the incident and scattered propagation directions defined by $\phi^{\prime}$ and $\phi$, respectively. The heterogeneities considered here are oriented with transverse isotropy such that $\stackrel{P}{\underline{P}}$ is a function only of $\Phi=\phi-\phi^{\prime}$.

The spatial Fourier transforms of the specific two-point correlation functions, $\widetilde{W}$, are defined in terms of the incident and scattered wave types and directions as ${ }^{10}$

$$
\begin{aligned}
& \widetilde{W}_{e e}(\Phi)=\widetilde{W}\left(\hat{\mathbf{p}} p_{e}^{0}-\hat{\mathbf{s}} p_{e}^{0}\right), \quad \widetilde{W}_{e s}(\Phi)=\widetilde{W}\left(\hat{\mathbf{p}} p_{e}^{0}-\hat{\mathbf{s}} p_{s}^{0}\right), \\
& \widetilde{W}_{e f}(\Phi)=\widetilde{W}\left(\hat{\mathbf{p}} p_{e}^{0}-\hat{\mathbf{s}} p_{f}^{0}\right), \\
& \widetilde{W}_{s e}(\Phi)=\widetilde{W}\left(\hat{\mathbf{p}} p_{s}^{0}-\hat{\mathbf{s}} p_{e}^{0}\right), \quad \widetilde{W}_{s s}(\Phi)=\widetilde{W}\left(\hat{\mathbf{p}} p_{s}^{0}-\hat{\mathbf{s}} p_{s}^{0}\right), \\
& \widetilde{W}_{s f}(\Phi)=\widetilde{W}\left(\hat{\mathbf{p}} p_{s}^{0}-\hat{\mathbf{s}} p_{f}^{0}\right), \\
& \widetilde{W}_{f e}(\Phi)=\widetilde{W}\left(\hat{\mathbf{p}} p_{f}^{0}-\hat{\mathbf{s}} p_{e}^{0}\right), \quad \widetilde{W}_{f s}(\Phi)=\widetilde{W}\left(\hat{\mathbf{p}} p_{f}^{0}-\hat{\mathbf{s}} p_{s}^{0}\right), \\
& \widetilde{W}_{f f}(\Phi)=\widetilde{W}\left(\hat{\mathbf{p}} p_{f}^{0}-\hat{\mathbf{s}} p_{f}^{0}\right) .
\end{aligned}
$$

As one might expect, the scattering matrix $\underline{\underline{P}}$ is related to the intensity attenuation matrix, $\underset{=}{\kappa}$. It is easily shown that

$$
\begin{aligned}
& \kappa_{e}=2 \alpha_{e}=\int_{0}^{2 \pi}\left[P_{11}(\Phi)+P_{21}(\Phi)+P_{31}(\Phi)\right] d \Phi, \\
& \kappa_{s}=2 \alpha_{s}=\int_{0}^{2 \pi}\left[P_{12}(\Phi)+P_{22}(\Phi)+P_{32}(\Phi)\right] d \Phi, \\
& \kappa_{f}=2 \alpha_{f}=\int_{0}^{2 \pi}\left[P_{13}(\Phi)+P_{23}(\Phi)+P_{33}(\Phi)\right] d \Phi,
\end{aligned}
$$

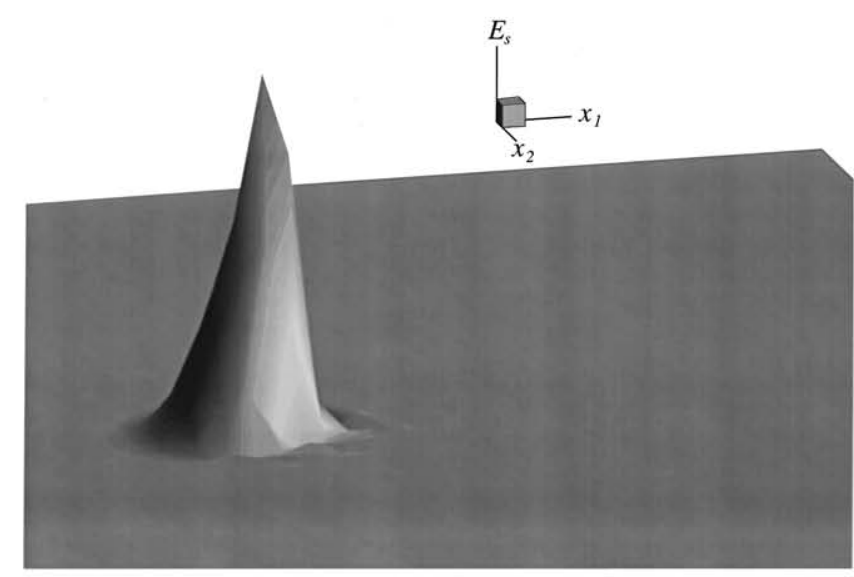

(a)

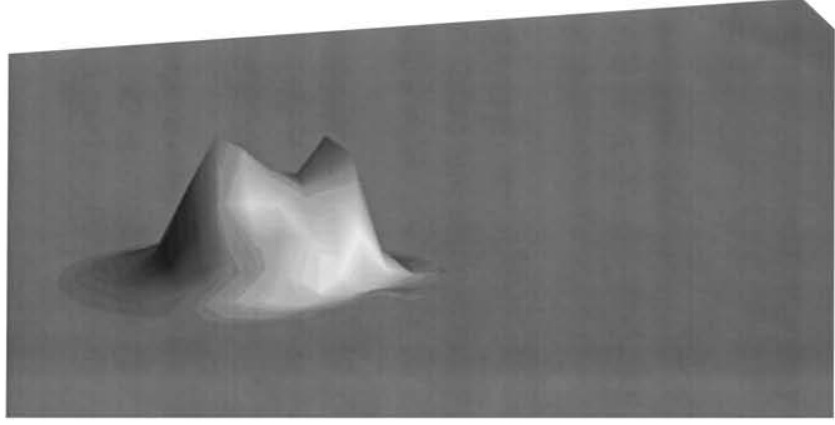

(b)

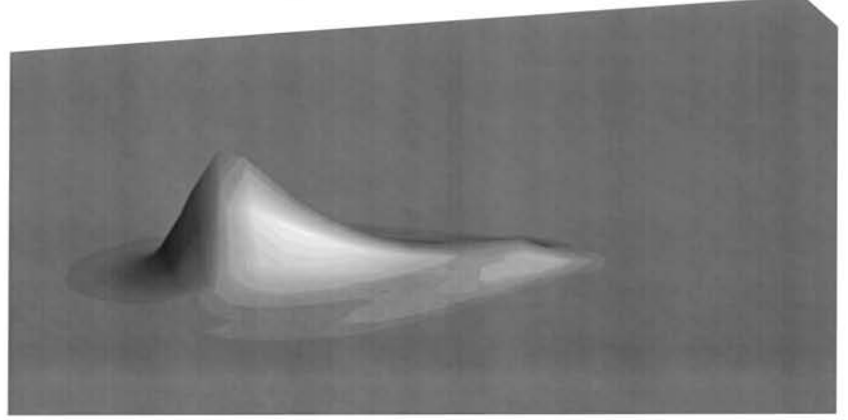

(c)

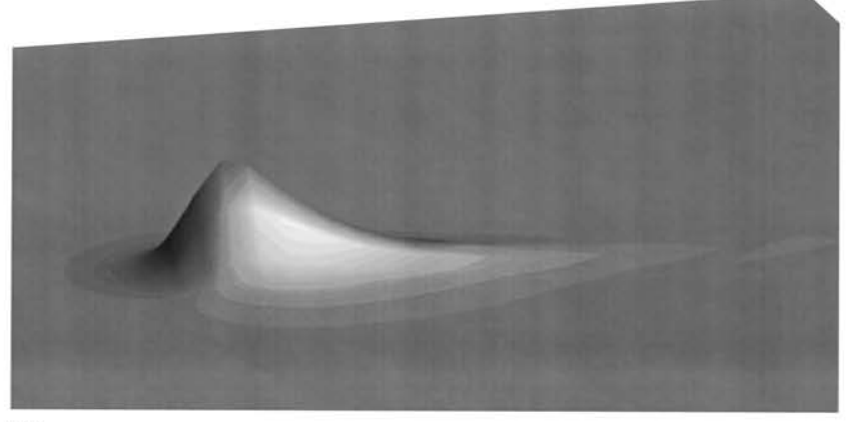

(d)

FIG. 3. Diffuse shear energy density, $E_{s}$, at $\widetilde{t}=1,2,4,7$ extensional meanfree times, respectively (a)-(d), to an incident extensional wave launched in the $\hat{x}_{1}$ direction. The shear energy density has been scaled by a factor of 2.86 when compared with the extensional energy density of Fig. 2.

as expected. ${ }^{20}$ Should the heterogeneities have internal degrees of freedom, such as those considered by the recent fuzzy structures literature, ${ }^{22-24}$ an additional dissipative matrix will arise which will account for those losses. ${ }^{10}$ 
The energy density is given in terms of the Stokes parameters by

$$
\begin{aligned}
E(\mathbf{r}, t)= & \int_{0}^{2 \pi}\left[I_{e}(\mathbf{r}, t, \phi) / c_{p}+I_{s}(\mathbf{r}, t, \phi) / c_{s}\right. \\
& \left.+I_{f}(\mathbf{r}, t, \phi) / 2 c_{f}\right] d \phi .
\end{aligned}
$$

The energy flux vector (called the intensity by Langley ${ }^{4,5}$ ) is similarly given by

$$
\begin{aligned}
\mathbf{F}(\mathbf{r}, t)= & \int_{0}^{2 \pi}\left\{I_{e}(\mathbf{r}, t, \phi)+I_{s}(\mathbf{r}, t, \phi)+I_{f}(\mathbf{r}, t, \phi)\right\} \\
& \times\{\hat{\mathbf{x}} \cos \phi+\hat{\mathbf{y}} \sin \phi\} d \phi .
\end{aligned}
$$

In the diffusion limit, the energy density and energy flux vector are further found to be related by $\mathbf{F}=-D \nabla E$, where $D$ is the appropriate diffusivity.

Equation (22) defines the total intensity, both coherent and diffuse. The interest here is primarily in the diffuse intensity. An equation for the diffuse intensity alone can also be derived. The total Stokes vector is first separated into its coherent and diffuse parts as

$$
\underline{I}=\underline{I}_{c}+\underline{I}_{d} .
$$

The coherent intensity, $\underline{I}_{c}$, is the solution of Eq. (22) with the integral term removed. For a point excitation of all three wave types the coherent solution is given by

$$
\underline{I}_{c}=\left\{\begin{array}{l}
I_{e}^{0} e^{-c_{p} \kappa_{e} t} \delta^{2}\left(\mathbf{r}-c_{p} t \hat{\mathbf{p}}\right) \delta\left(\phi-\phi_{e}^{0}\right) \\
I_{s}^{0} e^{-c_{s} \kappa_{s} t} \delta^{2}\left(\mathbf{r}-c_{s} t \hat{\mathbf{p}}\right) \delta\left(\phi-\phi_{s}^{0}\right) \\
I_{f}^{0} e^{-2 c_{f} \kappa_{f} t} \delta^{2}\left(\mathbf{r}-2 c_{f} t \hat{\mathbf{p}}\right) \delta\left(\phi-\phi_{f}^{0}\right)
\end{array}\right\},
$$

where $I_{e}^{0}, I_{s}^{0}$, and $I_{f}^{0}$ are the incident extensional, shear, and flexural intensities, launched in the respective directions $\phi_{e}^{0}$, $\phi_{s}^{0}$, and $\phi_{f}^{0}$.

Substitution of Eq. (30) into the SARTE results in an equation governing the diffuse intensity only

$$
\begin{aligned}
\underline{\underline{\underline{\mathbf{p}}}} \cdot \nabla \underline{I}(\mathbf{r}, t, \phi)+\underline{\underline{c}} \underline{\underline{\kappa}} \underline{\underline{I}}(\mathbf{r}, t, \phi)+\frac{\partial}{\partial t} \underline{I}(\mathbf{r}, t, \phi) \\
=\int_{0}^{2 \pi} \underline{\underline{c}} \underline{\underline{P}}\left(\phi, \phi^{\prime}\right) \underline{I}\left(\mathbf{r}, t, \phi^{\prime}\right) d \phi^{\prime} \\
+\underline{\Psi}_{e}\left(\phi, \phi_{e}^{0}\right) e^{-c_{p} \kappa_{e} t} \delta^{2}\left(\mathbf{r}-c_{p} t \hat{\mathbf{p}}_{e}^{0}\right) \\
+\underline{\Psi}_{s}\left(\phi, \phi_{s}^{0}\right) e^{-c_{s} \kappa_{s} t} \delta^{2}\left(\mathbf{r}-c_{s} t \hat{\mathbf{p}}_{s}^{0}\right) \\
+ \\
+\underline{\Psi}_{f}\left(\phi, \phi_{f}^{0}\right) e^{-2 c_{f} \kappa_{f} t} \delta^{2}\left(\mathbf{r}-2 c_{f} t_{\mathbf{p}_{f}^{0}}^{0}\right)
\end{aligned}
$$

The three source terms at the end of Eq. (32) are the coherent energies from each of the incident propagation types that have singly scattered into diffuse energy of all propagation-

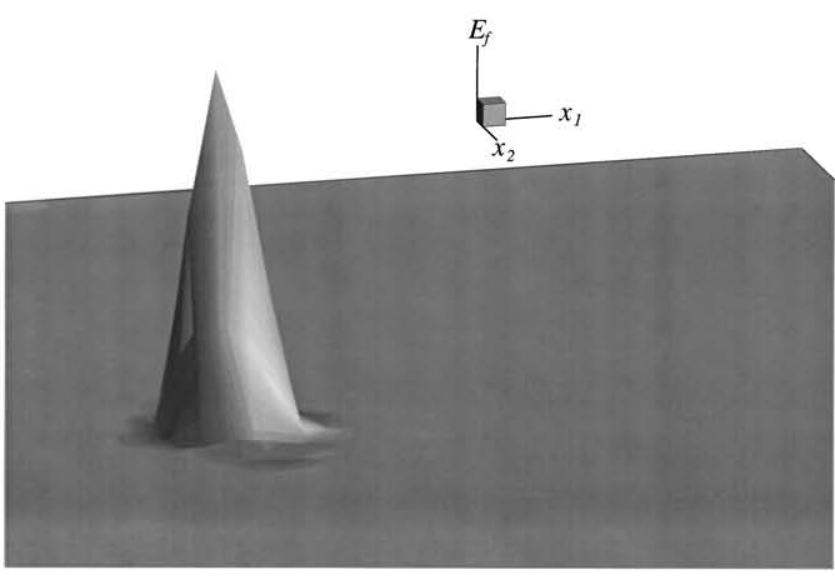

(a)

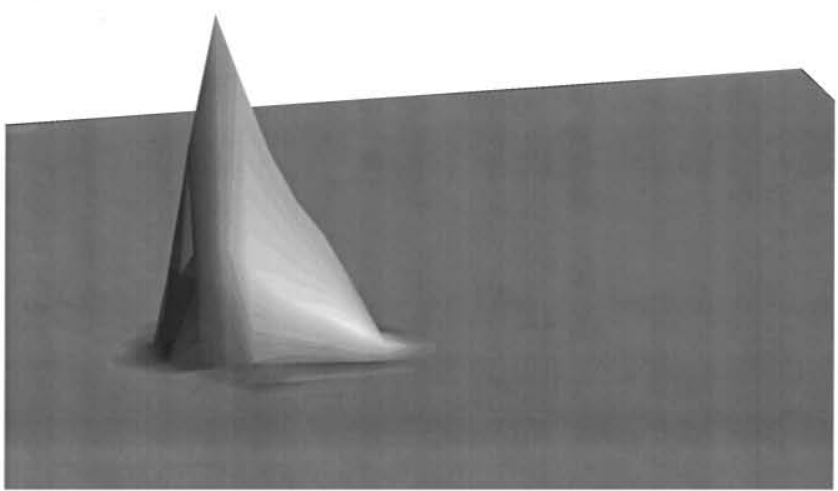

(b)

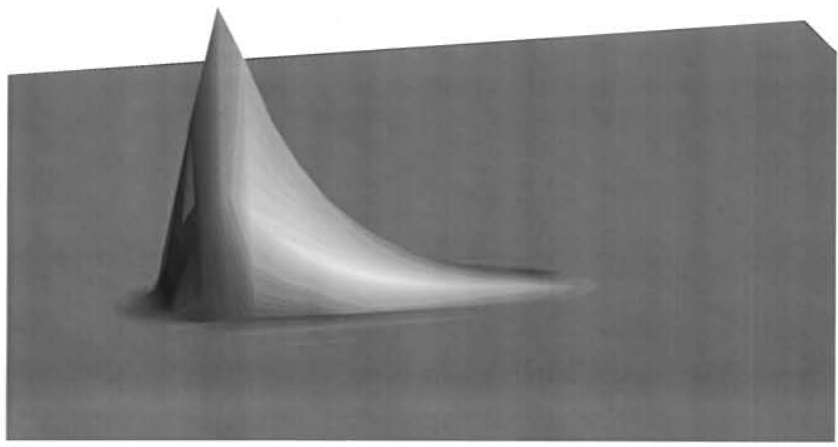

(c)

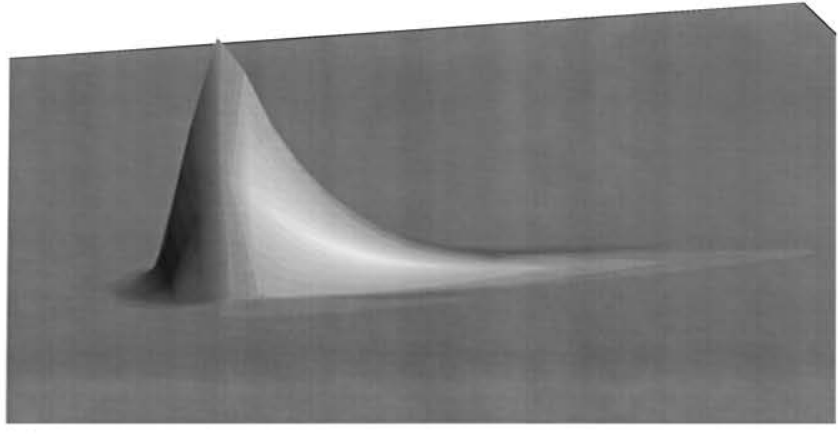

(d)

FIG. 4. Diffuse flexural energy density, $E_{f}$, at $\widetilde{t}=1,2,4,7$ extensional meanfree times, respectively (a)-(d), to an incident extensional wave launched in the $\hat{x}_{1}$ direction. The flexural energy density has been scaled by a factor of 171 when compared with the extensional energy density of Fig. 2.

types. Their amplitudes contain the scattering functions of the scattering matrix $\stackrel{\underline{P}}{P}$ and are given by 


$$
\begin{aligned}
& \underline{\Psi}_{e}\left(\phi, \phi_{e}^{0}\right)=I_{e}^{0}\left\{\begin{array}{l}
c_{p} P_{11}\left(\phi, \phi_{e}^{0}\right) \\
c_{s} P_{21}\left(\phi, \phi_{e}^{0}\right) \\
2 c_{f} P_{31}\left(\phi, \phi_{e}^{0}\right)
\end{array}\right\}, \\
& \underline{\Psi}_{s}\left(\phi, \phi_{s}^{0}\right)=I_{s}^{0}\left\{\begin{array}{l}
c_{p} P_{12}\left(\phi, \phi_{s}^{0}\right) \\
c_{s} P_{22}\left(\phi, \phi_{s}^{0}\right) \\
2 c_{f} P_{32}\left(\phi, \phi_{s}^{0}\right)
\end{array}\right\}, \\
& \Psi_{f}\left(\phi, \phi_{f}^{0}\right)=I_{f}^{0}\left\{\begin{array}{l}
c_{p} P_{13}\left(\phi, \phi_{f}^{0}\right) \\
c_{s} P_{23}\left(\phi, \phi_{f}^{0}\right) \\
2 c_{f} P_{33}\left(\phi, \phi_{f}^{0}\right)
\end{array}\right\} .
\end{aligned}
$$

\section{SOLUTIONS OF THE SARTE}

Simple closed-form solutions of radiative transfer equations are not known for general scattering matrices. ${ }^{15,16}$ Therefore, numerical solutions are often used for their solution. The case of an incident extensional point source launched in the $\hat{\mathbf{x}}_{1}$ direction is now examined. The SARTE for this problem is given by Eq. (32) above with the shear and flexural incident diffuse sources set to zero. Thus, we seek the solution of

$$
\begin{aligned}
& \underline{\underline{c} \hat{\mathbf{p}}} \cdot \boldsymbol{\nabla} \underline{I}(\mathbf{r}, t, \phi)+\underline{\underline{c}} \underline{\underline{\kappa}} \underline{\underline{I}}(\mathbf{r}, t, \phi)+\frac{\partial}{\partial t} \underline{I}(\mathbf{r}, t, \phi) \\
& =\int_{0}^{2 \pi} \underline{\underline{c}} \underline{\underline{P}}\left(\phi, \phi^{\prime}\right) \underline{I}\left(\mathbf{r}, t, \phi^{\prime}\right) d \phi^{\prime} \\
& +\underline{\Psi}_{e}(\phi, 0) e^{-c_{p} \kappa_{e} t} \delta^{2}\left(\mathbf{r}-c_{p} t \hat{\mathbf{x}}_{1}\right) .
\end{aligned}
$$

Equation (34) is first spatially Fourier transformed giving

$$
\begin{gathered}
-i \underline{\underline{c}}(\hat{\mathbf{p}} \cdot \Delta) \underline{I}(\Delta, t, \phi)+\underline{\underline{c}} \underline{\underline{\kappa}} \underline{\underline{I}}(\Delta, t, \phi)+\frac{\partial}{\partial t} \underline{I}(\Delta, t, \phi) \\
=\int_{0}^{2 \pi} \underline{\underline{c}} \underline{\underline{P}}\left(\phi, \phi^{\prime}\right) \underline{I}\left(\Delta, t, \phi^{\prime}\right) d \phi^{\prime} \\
\quad+\underline{\Psi}_{e}(\phi, 0) e^{-c_{p} \kappa_{e} t} e^{i c c_{p} t\left(\Delta \cdot \hat{\mathbf{x}}_{1}\right)},
\end{gathered}
$$

where $\Delta$ is the outer wave vector and spatial transform parameter (with components $\Delta_{1}$ and $\Delta_{2}$ in the $\hat{x}_{1}$ and $\hat{x}_{2}$ directions). The integral term of Eq. (35) is simplified by expanding the $\phi$ dependencies of $\underline{I}, \underline{P}$, and $\underline{\Psi}_{e}$ in terms of Fourier series as

$$
\begin{aligned}
& \underline{\underline{P}\left(\phi, \phi^{\prime}\right)}=\sum_{l=-N}^{+N} \underline{\underline{P}}_{l} e^{-i l\left(\phi-\phi^{\prime}\right)}, \\
& \underline{I}(\Delta, t, \phi)=\sum_{j=-N}^{+N} \underline{I}_{j}(\Delta, t) e^{i j \phi}, \\
& \underline{\Psi}_{e}(\phi)=\sum_{j=-N}^{+N} \underline{\Psi}_{e j} e^{i j \phi} .
\end{aligned}
$$

From the definition of the energy density given by Eq. (28), we see that the $j=0$ component of the $\underline{I}$ expansion is used to calculate the energy density.

These expansions are substituted into Eq. (35). The orthogonality of the Fourier terms removes the integral. The resulting equation is then successively multiplied by $e^{-i l \phi}$, for $l=-N \cdots+N$, and integrated over the unit circle. The result is a set of $6 N+3$ coupled ordinary differential equations

$$
\begin{aligned}
{[\underline{\underline{c}} \underline{\underline{\kappa}}} & \left.-2 \pi \underline{\underline{c}} \underline{\underline{P}}_{j}+\frac{d}{d t}\right] \underline{I}_{j}(\Delta, t)-\left[\underline{\underline{c}}\left(\Delta_{1}-i \Delta_{2}\right) i / 2\right] \underline{I}_{j-1}(\Delta, t) \\
& -\left[\underline{\underline{c}}\left(\Delta_{1}+i \Delta_{2}\right) i / 2\right] \underline{I}_{j+1}(\Delta, t) \\
= & \underline{\Psi}_{e j} e^{-c_{p} t\left(\kappa_{e}-i \Delta_{1}\right)}, \quad \forall j=-N \cdots+N .
\end{aligned}
$$

The above coupled, time-dependent ODEs can be easily solved at discrete values of the outer wave number components. A number of solution methods are possible with the constraint that the diffuse intensities are zero at $t=0$. Once the solution to Eq. (37) has been obtained in the spatial Fourier transform domain, an inverse fast Fourier transform can be used to return the solution to the $\left(x_{1}, x_{2}\right)$ domain.

\section{RESULTS FOR DELTA-CORRELATED SPRINGS}

A simple example is now presented in order to illustrate a result typical of Eq. (34). The case considered previously of heterogeneities in the form of delta-correlated linear springs attached to a rigid ground ${ }^{10}$ is examined in the context of diffuse energy propagation. This type of heterogeneity does not have internal degrees of freedom. Thus, the attached impedances act as lossless scatterers which redistribute the diffuse energy between the propagation modes and directions. These scatterers do not remove energy from the plate. For this case the scattering functions, $\xi(\Phi)$, used in the definition of the scattering matrix [Eq. (24)], are very simple and given by ${ }^{10}$

$$
\begin{aligned}
& \xi_{e e}(\Phi)=\sigma \bar{k}^{2}\left(1+2 \cos ^{2} \Phi\right) / 15 A, \\
& \xi_{e s}(\Phi)=\xi_{s e}(\Phi)=\sigma \vec{k}^{2}\left(1+2 \sin ^{2} \Phi\right) / 15 A, \\
& \xi_{e f}(\Phi)=\xi_{f e}(\Phi)=\sigma \vec{k}^{2} / 15 A, \\
& \xi_{s s}(\Phi)=\sigma \vec{k}^{2}\left(1+2 \cos ^{2} \Phi\right) / 15 A, \\
& \xi_{s f}(\Phi)=\xi_{f s}(\Phi)=\sigma \vec{k}^{2} / 15 A, \quad \xi_{f f}(\Phi)=3 \sigma \vec{k}^{2} / 15 A,
\end{aligned}
$$

where $\bar{k}$ is the dimensionless average spring stiffness, $\sigma$ is the number density of the springs, and $A$ is the plate area. The assumption that the spring positions are delta correlated implies that $\widetilde{W}(\Phi)=A /(2 \pi)^{2}$ for each scattering type.

As before, parameters are chosen for this example which are relevant to submerged thin shells. A dimensionless excitation frequency, $\boldsymbol{\varpi}=\omega h / c_{p}$, is defined which is unity for frequencies of the order of the coincidence frequency. Our interest is in frequencies near the "ring" frequency, where the cylinder's circumference fits one extensional wavelength. In this case, $\omega_{\text {ring }}=c_{p} / R$ with $R$ the cylinder radius. Thus assuming $R / h=100$ we use an excitation frequency, $\varpi=0.01$. The total added stiffness is assumed to equal the breathing mode stiffness for which $\sigma \bar{k}=10^{-4} / h^{2} .{ }^{10}$ Finally, the plate is assumed to be a 5 -cm-thick steel plate with spring density, $\sigma=1.15$ springs per square meter which implies that $\bar{k}=0.035$. These density and stiffness parameters result in significant scattering attenuations. ${ }^{10}$ 
The numerical scheme given in Sec. IV was used to reduce the SARTE, Eq. (34), to the system of coupled ODEs given by Eq. (37). Equation (37) was then converted to an eigenvalue problem governing the vector $\mathbf{I}=\left\{\mathbf{I}_{-\mathrm{N}}, \ldots\right.$, $\left.\mathrm{I}_{-0}, \ldots, \mathrm{I}_{+\mathrm{N}}\right\}^{\mathrm{T}}$, composed of the Fourier components of the specific intensities. The eigenvalues and eigenvectors describe the time domain evolution of $\mathbf{I}$. The eigenvalue problem was solved with $N=9$ and with 64 Fourier components for both outer wave vector directions. The solutions, obtained at discrete values of $\Delta_{1}$ and $\Delta_{2}$, provided time-wave vector domain solutions which were then numerically inverse fast Fourier transformed back to the time-space domain. Results of the extensional, shear, and flexural diffuse energy densities, $E_{e}, E_{s}$, and $E_{f}$ as a function of space and time are presented. The energy densities shown are obtained from the corresponding specific intensities according to Eq. (28). The results are shown at times given in terms of a dimensionless time, $\widetilde{t}=t c_{p} \kappa_{e}$, such that time is measured in units of the mean-free time of an extensional wave before scattering.

Figure 2 shows the evolution of the diffuse extensional energy density, $E_{e}$, at times $\tilde{t}=1,2,4,7$. A section of the plate near the origin is shown. The incident extensional wave has been launched at the origin in the $x_{1}$ direction. Figure 2(a) is the result after one extensional mean-free time, $\tilde{t}=1$. The large peak seen in Fig. 2(a) is the singly scattered energy. The diffuse energy can be imagined as a sum of multiple scattering events. At early times, single scattering will dominate the diffuse energy. This concept is evident in Fig. 2(a). Most of the diffuse energy at this early time has been scattered only once. The trailing envelope is the remaining scattered energy. In Fig. 2(b), the diffuse extensional energy at $\widetilde{t}=2$, the singly scattered energy is much smaller and is a smaller fraction of the total diffuse energy. The singly scattered energy moves at the speed of the incident intensity and decays exponentially. In Fig. 2(c), the result at four extensional mean-free times, the diffuse extensional energy is spatially much smoother. The singly scattered energy is now barely visible - only a small fraction of the total energy is in the singly scattered form. The diffuse energy is now dominated by energy that has scattered two or more times. The result at $\widetilde{t}=7$, shown in Fig. 2(d), shows little evidence of the singly scattered energy. Most of the extensional energy now is contained in the slowly spreading diffuse envelope which will become more isotropic as time progresses.

The evolution of the diffuse shear energy density, $E_{s}$, is shown in Fig. 3 for the same time steps of Fig. 2, $\widetilde{t}=1,2,4,7$. However, the amplitude scale is different from Fig. 2. As previously noted, the energy scattered in such a system is redistributed into all available propagation modes in such a way that the more densely packed modes receive more energy. ${ }^{10}$ The modal density of the shear waves is higher than the modal density of the extensional waves. Thus, the incident extensional wave will lose more energy into the shear wave type than into the extensional wave type. Therefore, the diffuse shear energy shown in Fig. 3 has been scaled by the modal density ratio, $n_{s}(\omega) / n_{e}(\omega)=c_{p}^{2} / c_{s}^{2}$, which, for the parameters chosen, is 2.86 . The evolution of the diffuse shear energy is similar to that of the extensional.
The singly scattered energy (extensional to shear) dominates at early times and becomes less important at later times.

Finally, the diffuse flexural energy evolution is shown in Fig. 4. The flexural energy, $E_{f}$, has also been scaled by the modal density ratio, $n_{f}(\omega) / n_{e}(\omega)=c_{p}^{2} / 2 c_{f}^{2}$. However, in this case, for the inner frequency chosen, the scaling is by a factor of 171 . The majority of the energy in the incident extensional wave is scattered into the flexural wave type. This result is also evident in the "tail" immediately behind the propagating source. The extensional source sheds a large amount of its energy immediately into the more densely packed flexural degrees of freedom.

This diffuse energy propagation can be examined for very late times as well. The energy in each propagation type is seen to slowly spread outward from the center of the plate becoming more isotropic. At late times or far from the source, the appropriate equipartitioning of energy is also observed as expected. ${ }^{9}$

\section{DISCUSSION}

The propagation and scattering of flexural and membrane waves on a heterogeneous plate has been formulated using radiative transfer theory. The structural acoustics radiative transfer equation (SARTE) was derived from consideration of an appropriately averaged wave equation. The parameters of the SARTE are given in terms of the statistics of the attached heterogeneities. The SARTE was derived using the assumption of weak scattering which is not expected to be very restrictive, but does not predict the development of Anderson localization.

However, the results for the simple example considered are very illustrative. The launched extensional wave was seen to lose its energy, because of scattering, into other directions and propagation modes. The high modal density of the flexural degrees of freedom acted as an energy sink to the majority of the incident energy deposited in extensional form. The diffuse energy was seen to evolve from early times, when it was dominated by the singly scattered energy, to late times, when the energy became more uniformly distributed and isotropic. The approach to the limit of energy equipartitioning between the extensional, shear, and flexural mode types at late times was also observed as expected.

The above analysis provides a methodology that can now be used for more complex structures that include spatially varying curvature and more complex attachments. Energy losses into a surrounding fluid and losses due to internal degrees of freedom must also be included in order to model more realistic structures.

\section{ACKNOWLEDGMENT}

This work was sponsored by the Office of Naval Research, Grant No. N00014-94-0855.

\footnotetext{
${ }^{1}$ R. H. Lyon, Statistical Energy Analysis of Dynamical Systems: Theory and Applications (MIT, Cambridge, MA, 1975).

${ }^{2}$ R. H. Lyon and G. Maidanik, "Power flow between linearly coupled oscillators," J. Acoust. Soc. Am. 34, 623-639 (1962).

${ }^{3}$ R. H. Lyon, "Statistical analysis of power injection and response in structures and rooms,"' J. Acoust. Soc. Am. 45, 545-565 (1969).
} 
${ }^{4}$ R. S. Langley, "A wave intensity technique for the analysis of high frequency vibrations,"' J. Sound Vib. 159, 483-502 (1992).

${ }^{5}$ R. S. Langley, "On the vibrational conductivity approach to high frequency dynamics for two-dimensional structural components," J. Sound Vib. 182, 637-657 (1995).

${ }^{6}$ R. L. Weaver, "Diffuse waves in finite plates," J. Sound Vib. 94, 319335 (1984).

${ }^{7}$ R. L. Weaver, "Laboratory studies of diffuse waves in plates," J. Acoust. Soc. Am. 79, 919-923 (1986).

${ }^{8}$ R. L. Weaver, "Diffuse elastic waves at a free surface,' J. Acoust. Soc. Am. 78, 131-136 (1985).

${ }^{9}$ R. L. Weaver, "Diffuse waves on submerged thin shells," J. Acoust. Soc. Am. 95, 857-865 (1994).

${ }^{10}$ J. A. Turner and R. L. Weaver, "Coupling and attenuation of waves in plates by randomly distributed attached impedances," J. Acoust. Soc. Am. 99, 2167-2175 (1996).

${ }^{11}$ S. A. Rybak, "Waves in a plate containing random inhomogeneities," Sov. Phys. Acoust. 17, 345-349 (1972).

${ }^{12} \mathrm{~S}$. A. Rybak, "Randomly coupled flexural and longitudinal vibrations of plates," Sov. Phys. Acoust. 18, 76-79 (1972).

${ }^{13}$ U. Frisch, "Wave propagation in random media," in Probabilistic Methods in Applied Mathematics, edited by A. T. Bharucha-Reid (Academic, New York, 1968), Vol. 1, pp. 75-198.

${ }^{14}$ R. L. Weaver, "Diffusivity of ultrasound in polycrystals," J. Mechan. Phys. Solids 38, 55-86 (1990).

${ }^{15}$ A. Ishimaru, Wave Propagation and Scattering in Random Media (Academic, New York, 1978), Vols. 1 and 2.
${ }^{16}$ L. Tsang, J. A. Kong, and R. T. Shin, Theory of Microwave Remote Sensing (Wiley, New York, 1985).

${ }^{17}$ F. C. Karal and J. B. Keller, "Elastic, electromagnetic, and other waves in random media," J. Math. Phys. 5, 537-547 (1964).

${ }^{18}$ R. L. Weaver, "Anderson localization of ultrasound," Wave Motion 12, 129-142 (1990).

${ }^{19}$ E. N. Economou, Green's Functions in Quantum Physics (SpringerVerlag, Berlin, 1979).

${ }^{20}$ J. A. Turner and R. L. Weaver, "Radiative transfer and multiple scattering of diffuse ultrasound in polycrystalline media," J. Acoust. Soc. Am. 96, 3675-3683 (1994).

${ }^{21}$ N. M. Ozisik, Radiative Transfer and Interactions with Conduction and Convection (Pergamon, Oxford, 1973).

${ }^{22} \mathrm{C}$. Soize, "Modeling and numerical method in the medium frequency range for vibroacoustic predictions using theory of structural fuzzy," J. Acoust. Soc. Am. 92, 2365 (1992).

${ }^{23}$ A. D. Pierce, V. W. Sparrow, and D. A. Russell, "Fundamental structuralacoustic idealizations for structures with fuzzy internals," J. Vib. Acoust. Trans. ASME 117, 339-348 (1995).

${ }^{24}$ A. D. Pierce, "Mass per unit natural frequency as a descriptor of internal fuzzy structure," Boston University Technical Report No. AM-94-003, June 1994. "Influence of structural wave dispersion on scattering patterns, with application to scattering from structures with fuzzy internal," Boston University Technical Report No. AM-94-006, July, 1994. "Mechanical foundations for a theory of fuzzy structures," Boston University Technical Report No. AM-95-003, January 1995. 